\title{
A Novel Rodent Model Modifying Perioperative Temperature and Humidity during Bowel Surgery and Mimicking Laparoscopic Conditions
}

\author{
Torben Glatz", Gabriel Seifert, Philipp A. Holzner, S. Chikhladze, Birte Kulemann, Olivia Sick, \\ Jens Höppner, Ulrich Theodor Hopt, Goran Marjanovic \\ Department of General and Visceral Surgery, Albert Ludwigs University, Freiburg, Germany \\ Email: *Torben.Glatz@uniklinik-freiburg.de
}

Received May 20, 2012; revised June 10, 2012; accepted June 25, 2012

\begin{abstract}
Laparoscopic surgery seems to have a general physiological benefit compared to conventional procedures in several clincal studies especially regarding intestinal anastomotic healing. Reliable experimental data concerning the particular mode of beneficial action are lacking. Clinical studies will not be able to identify the variables responsible for this effect. To establish a feasible, standardized experimental model to investigate variables such as humidity and perioperative room temperature, we employed an incubator designed for neonatal care. This allows individual manipulation and observation of above mentioned variables. Our initial results show that creating intestinal anastomoses in the incubator is safe and easy to perform, creating a valuable possibility to influence perioperative conditions for experimental and clinical research.
\end{abstract}

Keywords: Intestinal Anastomosis; Anastomotic Leak; Laparoscopy; Incubator; Rat

\section{Introduction}

During the last decade, laparoscopic techniques have become more and more popular in most surgical fields. Today minimal invasive bowel resections have been established as a standard procedure in most hospitals. The laparoscopic approach appears to be a safe option in benign and malignant diseases [1-4], and clinical analyses have even shown an improved outcome of laparoscopic bowel resections with primary intestinal anastomoses compared to open procedures [5].

Little is known about the mechanisms of the effects of laparoscopic surgery itself on anastomotic healing. First of all, the selection of patients suitable for a laparoscopic approach must be considered. In patients with severe comorbidities, such as liver cirrhosis or chronic renal failure, a septic condition or a history of abdominal surgery, an open approach is often preferred over a minimal invasive operation. These factors as well as technical aspects, including different techniques of fashioning anastomoses, perioperative management and the level of experience of surgical staff may influence anastomotic healing [6,7] thus creating an inevitable bias of any clinical investigation of the safety and benefit of laparoscopically fashioned anastomoses [8-10].

"Corresponding author.
Several specific characteristics of the laparoscopic technique such as gas pressure [11-13], intraoperative temperature $[14,15]$ and humidity $[16,17]$ are currently being discussed but have not been adequately investigated regarding their impact on anastomotic healing due to the lack of a standardized experimental model.

However, ultimately only an appropriate animal model will allow systematic investigation of the altered intraoperative situation of laparoscopic surgery. A laparoscopic operation can be performed in large animals like pigs or sheep, but experiments are expensive, laborious and ethically disputable. In addition, the perioperative factors intraabdominal pressure, temperature, humidity and core temperature of the animal are difficult to control.

In the past, our group established a model of intestinal anastomoses in rats, providing an easy, accessible and inexpensive method of evaluating perioperative factors that can divert the course of normal anastomotic healing in either direction [18-23]. The aim of this study was to establish a module to mimic the conditions of minimal invasive surgery in an experimental rodent setting.

\section{Material and Methods}

\subsection{Experimental Groups and Operative Procedure}

Thirty male Wistar rats were randomized to three groups 
of ten rats each: group 1 (control) with standardized open procedure under normal operating room conditions at room temperature and low humidity, group 2 under incubator conditions with $30^{\circ} \mathrm{C}$ temperature and $60 \%$ humidity and group 3 under incubator conditions with $37^{\circ} \mathrm{C}$ temperature and $60 \%$ humidity (Table 1). The preoperative setting and the creation of a small bowel anastomosis was performed $15 \mathrm{~cm}$ proximal of Bauhin's valve after resection of $1 \mathrm{~cm}$ Ileum in a standardized setup as described before [22,24]. The environment created during operation for the different groups is shown in Table $\mathbf{1}$. The parameters chosen are exemplary to reveal some of the possibilities provided by the incubator.

\subsection{Incubator-Technical Aspects}

To create conditions comparable with a laparoscopic environment we employed an incubator originally designed for neonatal intensive care (Dräger 8000 IC Isolette (Figure 1), capable of creating surrounding temperatures between $0^{\circ} \mathrm{C}$ and $60^{\circ} \mathrm{C}$ and humidity of $15 \%$ to $95 \%$.

\subsection{Incubator-Preoperative Arrangements}

In preparation for surgery, the incubator needs a leadtime of about 30 minutes to create constant temperature and humidity. The rat was narcotized and transferred to the incubator after a stable environment had been estabwith $1.5 \%$ - 3\% Isofluran in Oxygen with a special mask.

Table 1. Study-design: small bowel anastomosis was performed in three groups of rats in different environment. The first group is the control group, group $2\left(30^{\circ} \mathrm{C} / 60 \%\right)$ and $3\left(37^{\circ} \mathrm{C} / 60 \%\right)$ are operated in the incubator at different temperature levels.

\begin{tabular}{cccc}
\hline Group & Procedure & Temperature & Humidity \\
\hline 1 & Open standard & Room temperature & Room climate \\
2 & Incubator & $30^{\circ} \mathrm{C}$ & $60 \%$ \\
3 & Incubator & $37^{\circ} \mathrm{C}$ & $60 \%$ \\
\hline
\end{tabular}

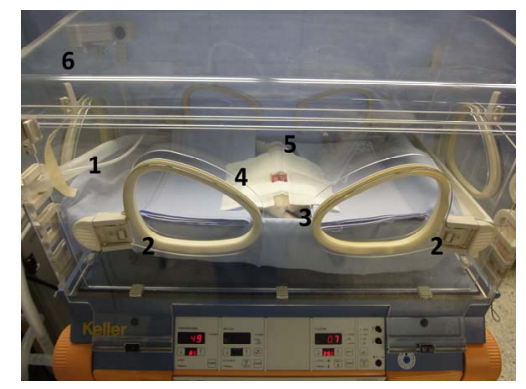

Employed for creating a unique environment of defined temperature and humidity for experimental surgery. 1) Ventilation; 2) Working access; 3) Temperature probe; 4) Aseptic coverage; 5) Narcotized rat; 6) Incubator.

Figure 1. The dräger 800 IC isolette. lished. The rodent was anesthetized during the operation. The procedure for anesthesia is analogous to the open operation. During the entire operation, the rat remained inside the incubator. The temperature probe and tube for the anesthetic gas were inserted through the working access.

\subsection{Incubator-Operative Aspects}

After laparotomy, small bowel resection and enteroanastomosis with single sutures (Prolene 8/0) were performed as described before [22,24]. The surgeon operates through the front working access (Figure 2). While anastomoses outside of the incubator can be performed in a sitting position, the surgeon has to stand to perform the procedure in the incubator. However, height and position of the incubator and the underlay can be adjusted to create a comfortable position for the surgeon.

While operating through the working access, the climate inside the incubator remains stable. The design of the device prevents a loss of humidity or heat. The body temperature of the narcotized rat was monitored through the entire procedure.

After completion of the small bowel anastomosis, the abdomen was closed and the rat was transferred back to the cage for postop follow-up.

The rats were kept in a controlled environment for 4 days with limited access to food and water. Rectal temperature and weight were taken daily.

On the fourth day, the animals were euthanized by intracardial injection of potassium. The abdomen was reopened and evaluated for relevant operative complications. The reoperation was performed outside the incubator.

\section{Results}

\subsection{Morbidity and Mortality}

During the four days of postoperative monitoring, we recorded two cases of anastomotic leakage, one in the

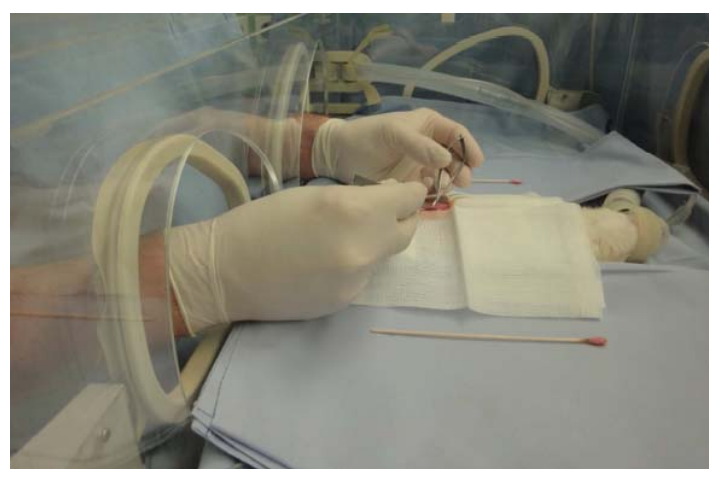

Laparotomy and small bowel anastomosis are performed entirely in an controlled environment created by the isolette.

Figure 2. Procedure performed in the incubator. 
control group and one in group $2\left(30^{\circ} \mathrm{C} / 60 \%\right)$. One rat in group $3\left(37^{\circ} \mathrm{C} / 60 \%\right)$ died of an unknown cause, most likely urosepsis. A postoperative ileus was not seen.

\subsection{Technical Aspects}

To secure comparable groups, perioperative data was taken (Table 2). Surgery time was almost identical among the groups, with approximately 44 minutes total operating time. Technically, the creation of the anastomosis was not different, but the surgeon must adapt his depth of view through the incubator barriers. In case of $60 \%$ humidity we noted slight condensation on the view pane, but this was not a problem for the operation. This was not seen in lower grades of humidity. Furthermore, it may be subjectively demanding for the surgeon to get along with a significantly higher ambient temperature and especially the high humidity.

\subsection{Perioperative Data}

The temperature of the rats was monitored during the operation. The animals being operated in the incubator showed an increase of up to $1^{\circ} \mathrm{C}$ while being kept in the incubator. The temperature dropped rapidly after the end of the procedure to normal levels. The core temperature postoperatively showed no difference among the groups, constantly staying at an average of $36^{\circ} \mathrm{C}$ to $37^{\circ} \mathrm{C}$. Fever $\left(>38^{\circ} \mathrm{C}\right)$ did not occur in any of the animals in the postoperative period.

The rats started at an approximate weight of $300 \mathrm{~g}$. All rats lost a significant amount of weight after the procedure, the weight loss averaging at roughly $10 \%$ of original bodyweight. There was no significant difference among the groups.

\section{Discussion}

The trauma induced by laparotomy and several hours of open surgery causes major changes in the patient's physiology. Systematic inflammatory response is triggered by the procedure, cytokine levels are elevated and immune function is suppressed. Clinical analyses have shown that patients after colectomy show significantly lower levels of CRP and interleukine- 6 as well as higher immune function of HLA-DR if treated laparoscopically
[25]. Retrospective analyses even indicate a lower rate of serious complications like anastomotic leak after laparoscopic surgery $[5,26]$, but systematic trials have failed to verify this effect [25]. These trials have limited value due to the low number of included patients and high numbers of distortive factors, like individual risk factors of the patient, technical factors, and experience of the surgical staff.

Laparoscopy itself changes the environment during surgery, influencing several factors that may explain a deviating outcome of minimal invasive Surgery, for example of intestinal anastomosis. The pneumoperitoneum during minimal invasive surgery creates constant pressure, humidity and temperature [13] and allows for less bacterial contamination [27,28]. Clinical and even experimental investigations stress the influence of these factors. Harmful effects of the intraoperative pneumoperitoneum on anastomotic healing have been shown in an experimental rodent model using laparoscopic technique [13]. Burst pressure in animals operated on laparoscopically depended on the gas pressure used.

Furthermore, there appears to be a an influence of the operating environment on the degree of intestinal adhesions and healing processes [29-31]. These results contradict the clinical findings reported by many groups $[5,26]$ and those of other experimental settings $[11,12]$.

To date, answers to the question whether laparoscopic surgery compared to an open procedure leads to significant changes in the patient's physiology and secures a better outcome and lower incidence of complications remains open. Only systematic experimental surgery will provide further insights. The minimal invasive approach changes three major factors: First there is less trauma, hence less systematic inflammation; second there is a constant overload pressure inside the abdomen for the time of surgery, built up by $100 \% \mathrm{CO}_{2}$; third there is a constant environment of body temperature and humidity $[11,15,25]$. The focus of our research lies in the specific mechanisms of anastomotic healing. Now we created a model that allows analysis of the particular effects of intraoperative humidity and temperature in different surgical fields.

Our model is the first approach to separately examine the impact of humidity and body temperature. While the

Table 2. Results of the performed operation: presented are the duration of the operation, the perioperative core temperature of the animals, the original weight and the total weight loss of the animals until the fourth day after anastomosis. There are no significant changes among the groups. All results are mean \pm SEM.

\begin{tabular}{|c|c|c|c|c|c|c|c|}
\hline Group & $\begin{array}{l}\text { OP duration } \\
\text { (min) }\end{array}$ & $\begin{array}{c}\text { Temperature directly } \\
\text { before OP }\left({ }^{\circ} \mathrm{C}\right)\end{array}$ & $\begin{array}{c}\text { Temperature directly } \\
\text { after OP }\left({ }^{\circ} \mathrm{C}\right)\end{array}$ & $\begin{array}{c}\text { Temperature } 1 \\
\text { day }\left({ }^{\circ} \mathrm{C}\right)\end{array}$ & $\begin{array}{c}\text { Temperature } 4 \\
\text { day }\left({ }^{\circ} \mathrm{C}\right)\end{array}$ & $\begin{array}{l}\text { Starting body } \\
\text { weight (g) }\end{array}$ & $\begin{array}{l}\text { Weightloss } 4 \text { days } \\
\text { after OP (\%) }\end{array}$ \\
\hline 1 control & $44.5( \pm 7.6)$ & $36.8( \pm 0.4)$ & $37.4( \pm 0.5)$ & $37.0( \pm 1.0)$ & $36.4( \pm 1.2)$ & $292( \pm 50.9)$ & $9( \pm 5.0)$ \\
\hline $230^{\circ} \mathrm{C} / 60 \%$ & $43.5( \pm 7.1)$ & $37.0( \pm 0.2)$ & $37.9( \pm 0.6)$ & $37.0( \pm 1.0)$ & $36.5( \pm 1.0)$ & $312.2( \pm 50.1)$ & $11( \pm 2.0)$ \\
\hline $337^{\circ} \mathrm{C} / 60 \%$ & $43.0( \pm 6.7)$ & $36.9( \pm 0.4)$ & $37.7( \pm 0.4)$ & $36.9( \pm 0.9)$ & $36.2( \pm 0.7)$ & $302.3( \pm 42.9)$ & $7( \pm 4.1)$ \\
\hline
\end{tabular}


rodents operated in the incubator experienced the same laparotomy as the control group and were not exposed to an intrabdominell pressure overload, we were able to control the surrounding temperature and humidity. Differences seen between the groups can be attributed specifically to these factors and experiments can be reproduced at will. In the laparoscopic rodent model described by Tytgat et al. [13], differentiation between the effects of humidity and temperature was not possible, and therefore the observed effects were attributed to differing gas pressure values. Our model allows us to adjust temperature between $0{ }^{\circ} \mathrm{C}$ and $60^{\circ} \mathrm{C}$ and humidity between $15 \%$ and $95 \%$, but there are physiological limits to the operative setting. Even the incubator is not able to create an unphysiological environment with low temperature and high humidity. Furthermore, the surface of the incubator will fog at very high humidity values, making the procedure difficult for the surgeon to perform. Nonetheless, a reasonable temperature scale of $20^{\circ} \mathrm{C}$ to $40^{\circ} \mathrm{C}$ with corresponding humidity can be examined and allows for an easy and safe experimental approach to mimic laparoscopic surgery.

While we employed the model to investigate anastomotic healing, it may well serve many other purposes, allowing closer observation of altered body physiology due to different levels of humidity and ambient temperature. The effects humidity and temperature and their role in diverting outcomes of open and laparoscopic surgery can be determined for any surgical procedure. In conclusion, we were able to show that operating in the incubator is feasible, fast and safe for the animals and the surgeon. Further experiments will follow.

\section{REFERENCES}

[1] E. Cotte, F. Mohamed, S. Nancey, Y. François, O. Glehen, B. Flourié, et al., "Laparoscopic Total Colectomy: Does the Indication Influence the Outcome?” The World Journal of Gastrointestinal Surgery, Vol. 3, No. 11, 2011, pp. 177-182.

[2] K. El Zarrok Elgazwi, I. Baca, L. Grzybowski and A. Jacks, "Laparoscopic Sigmoidectomy for Diverticulitis: A Prospective Study," Journal of the Society of Laparoendscopic Surgeons, Vol. 14, No. 4, 2010, pp. 469-475.

[3] D. W. Good, J. M. O’Riordan, D. Moran, F. B. Keane, E. Eguare, D. S. O’Riordain, et al., "Laparoscopic Surgery for Rectal Cancer: A Single-Centre Experience of 120 Cases," International Journal of Colorectal Disease, Vol. 26, No. 10, 2011, pp. 1309-1315. doi:10.1007/s00384-011-1261-1

[4] J. Royds, J. O’Riordan, E. Eguare, D. O’Riordain and P. Neary, "Laparoscopic Surgery for Complicated Diverticular Disease: A Single Centre Experience,” Colorectal Disease, 2011. doi:10.1111/j.1463-1318.2011.02924.x

[5] J. J. Luján, Z. H. Németh, P. A. Barratt-Stopper, R. Bustami, V. P. Koshenkov and R. H. Rolandelli, "Factors In- fluencing the Outcome of Intestinal Anastomosis," The American Journal of Surgery, Vol. 77, No. 9, 2011, pp. 1169-1175.

[6] J. W. T. Dekker, G. J. Liefers, J. C. A. de van Mol Otterloo, H. Putter and R. A. E. M. Tollenaar, "Predicting the Risk of Anastomotic Leakage in Left-Sided Colorectal Surgery Using a Colon Leakage Score,” Journal of Surgical Research, Vol. 166, No. 1, 2011, pp. e27-e34. doi:10.1016/j.jss.2010.11.004

[7] C. H. Richards, V. Campbell, C. Ho, J. Hayes, T. Elliott and M. Thompson-Fawcett, "Smoking Is a Major Risk Factor for Anastomotic Leak in Patients Undergoing Low Anterior Resection,” Colorectal Disease, Vol. 14, No. 5, 2011, pp. 628-633.

doi:10.1111/j.1463-1318.2011.02718.x

[8] M. A. Boccola, P. G. Buettner, W. M. Rozen, S. K. Siu, A. R. L. Stevenson, R. Stitz, et al., "Risk Factors and Outcomes for Anastomotic Leakage in Colorectal Surgery: A Single-Institution Analysis of 1576 Patients," World Journal of Surgery, Vol. 35, No. 1, 2011, pp. 186-195. doi:10.1007/s00268-010-0831-7

[9] M. A. Boccola, J. Lin, W. M. Rozen and Y. Ho, "Reducing Anastomotic Leakage in Oncologic Colorectal Surgery: An Evidence-Based Review,” Anticancer Research, Vol. 30, No. 2, 2010, pp. 601-607.

[10] D. H. Choi, J. K. Hwang, Y. T. Ko, H. J. Jang, H. K. Shin, Y. C. Lee, et al., "Risk Factors for Anastomotic Leakage after Laparoscopic Rectal Resection,” Journal of the Korean Society of Coloproctology, Vol. 26, No. 4, 2010, pp. 265-273.

[11] R. Rosch, M. Stumpf, K. Junge, D. Ardic, F. Ulmer and V. Schumpelick, "Impact of Pressure and Gas Type on Anastomotic Wound Healing in Rats," Langenbecks Archives of Surgery, Vol. 389, No. 4, 2004, pp. 261-266. doi:10.1007/s00423-004-0491-x

[12] M. Ulas, I. Ozer, M. Ercan, Y. B. Ozogul, E. B. Bostanci, T. T. Keklik, et al., "Effects of $\mathrm{CO}_{2}$ Pneumoperitoneum on Anastomotic Healing in Rats Receiving Preoperative 5-Fluorouracil Neoadjuvant Chemotherapy,” Journal of Investigative Surgery, Vol. 22, No. 6, 2009, pp. 413-418. doi:10.3109/08941930903410841

[13] S. H. A. J. Tytgat, G. T. Rijkers and D. C. van der Zee, "The Influence of the $\mathrm{CO}_{2}$ Pneumoperitoneum on a Rat Model of Intestinal Anastomosis Healing," Surgical Endoscopy, Vol. 26, No. 6, 2011, pp. 1642-1647. doi:10.1007/s00464-011-2086-2

[14] A. M. Kappas, K. Papadimitriou, M. Fatouros and D. Cassioumis, "Influence of Local Hyperthermia on the Healing of Small Intestinal Anastomoses in the Rat," British Journal of Surgery, Vol. 78, No. 7, 1991, p. 890. doi:10.1002/bjs.1800780742

[15] T. Shimizu, M. Maeta and S. Koga, "Influence of Local Hyperthermia on the Healing of Small Intestinal Anastomoses in the Rat," British Journal of Surgery, Vol. 78, No. 1, 1991, pp. 57-59. doi:10.1002/bjs.1800780119

[16] R. Corona, J. Verguts, R. Koninckx, K. Mailova, M. M. Binda and P. R. Koninckx, "Intraperitoneal Temperature and Desiccation during Endoscopic Surgery. Intraoperative Humidification and Cooling of the Peritoneal Cavity 
Can Reduce Adhesions," American Journal of Obstetrics \& Gynecology, Vol. 205, No. 4, 2011, pp. 392.e1-392.e7.

[17] D. W. Birch, N. Manouchehri, X. Shi, G. Hadi and S. Karmali, "Heated $\mathrm{CO}_{2}$ with or without Humidification for Minimally Invasive Abdominal Surgery,” Cochrane Database of Systematic Reviews, 2011, p. CD007821.

[18] J. Hoeppner, V. Crnogorac, G. Marjanovic, E. Jüttner, W. Karcz, H. Weiser, et al., "Small Intestinal Submucosa as a Bioscaffold for Tissue Regeneration in Defects of the Colonic Wall,” Journal of Gastrointestinal Surgery, Vol. 13, No. 1, 2009, pp. 113-119. doi:10.1007/s11605-008-0639-z

[19] J. Hoeppner, G. Marjanovic, P. Helwig, U. T. Hopt and T. Keck, "Extracellular Matrices for Gastrointestinal Surgery: Ex Vivo Testing and Current Applications,” World Journal of Gastroenterology, Vol. 16, No. 32, 2010, pp. 4031-4038. doi:10.3748/wjg.v16.i32.4031

[20] J. Hoeppner, B. Wassmuth, G. Marjanovic, S. Timme, U. T. Hopt and T. Keck, "Anastomotic Sealing by Extracellular Matrices (ECM) Improves Healing of Colonic Anastomoses in the Critical Early Phase," Journal of Gastrointestinal Surgery, Vol. 14, No. 6, 2010, pp. 977-986. doi:10.1007/s11605-010-1191-1

[21] J. Hoeppner, K. Willa, S. Timme, D. Tittelbach-Helmrich, U. T. Hopt, T. Keck, et al., "Reinforcement of Colonic Anastomoses with a Collagenous Double-Layer Matrix Extracted from Porcine Dermis," European Surgical Research, Vol. 45, No. 2, 2010, pp. 68-76. doi:10.1159/000318856

[22] G. Marjanovic, C. Villain, E. Juettner, A. Zur Hausen, J. Hoeppner, U. T. Hopt, et al., "Impact of Different Crystalloid Volume Regimes on Intestinal Anastomotic Stability," Annals of Surgery, Vol. 249, No. 2, 2009, pp. 181-185. doi:10.1097/SLA.0b013e31818b73dc

[23] G. Marjanovic, C. Villain, S. Timme, A. Zur Hausen, J. Hoeppner, F. Makowiec, et al., "Colloid vs. Crystalloid Infusions in Gastrointestinal Surgery and Their Different Impact on the Healing of Intestinal Anastomoses," International Journal of Colorectal Disease, Vol. 25, No. 4, 2010, pp. 491-498. doi:10.1007/s00384-009-0854-4

[24] G. Marjanovic, E. Jüttner, A. Zur Hausen, U. Theodor Hopt and R. Obermaier, "Ischemic Preconditioning Improves Stability of Intestinal Anastomoses in Rats,” In- ternational Journal of Colorectal Disease, Vol. 54, No. 8, 2009, pp. 975-981.

[25] A. F. A. Veenhof, M. S. Vlug, M. H. G. M. van der Pas, C. Sietses, D. L. van der Peet, E. S. M. de Lange-de Klerk, et al., "Surgical Stress Response and Postoperative Immune Function after Laparoscopy or Open Surgery with Fast Track or Standard Perioperative Care: A Randomized Trial,” Annals of Surgery, Vol. 255, No. 2, 2012, pp. 216-221. doi:10.1097/SLA.0b013e31824336e2

[26] M. Levack, D. Berger, P. Sylla, D. Rattner and L. Bordeianou, "Laparoscopy Decreases Anastomotic Leak Rate in Sigmoid Colectomy for Diverticulitis," Archives of Surgery, Vol. 146, No. 2, 2011, pp. 207-210. doi:10.1001/archsurg.2010.325

[27] Azadani, H. Jonsson, P. Park and M. Bergström, “A Randomized Trial Comparing Rates of Abdominal Contamination and Postoperative Infection among Natural Orifice Transluminal Endoscopic Surgery, Laparoscopic Surgery, and Open Surgery in Pigs," Gastrointestinal Endoscopy, Vol. 75, No. 4, 2012, pp. 849-855. doi:10.1016/j.gie.2011.11.039

[28] Y. Saida, J. Nagao, Y. Nakamura, Y. Nakamura, T. Enomoto, M. Katagiri, et al., "A Comparison of Abdominal Cavity Bacterial Contamination of Laparoscopy and Laparotomy for Colorectal Cancers,” Digestive Surgery, Vol. 25, No. 3, 2008, pp. 198-201. doi:10.1159/000140689

[29] M. M. Binda, C. R. Molinas, K. Mailova and P. R. Koninckx, "Effect of Temperature upon Adhesion Formation in a Laparoscopic Mouse Model,” Human Reproduction, Vol. 19, No. 11, 2004, pp. 2626-2632. doi:10.1093/humrep/deh495

[30] M. M. Binda, C. R. Molinas, P. Hansen and P. R. Koninckx, "Effect of Desiccation and Temperature during Laparoscopy on Adhesion Formation in Mice,” Fertility and Sterility, Vol. 86, No. 1, 2006, pp. 166-175. doi:10.1016/j.fertnstert.2005.11.079

[31] O. A. Elkelani, M. M. Binda, C. R. Molinas and P. R. Koninckx, "Effect of Adding More than 3\% Oxygen to Carbon Dioxide Pneumoperitoneum on Adhesion Formation in a Laparoscopic Mouse Model," Fertility and Sterility, Vol. 82, No. 6, 2004, pp. 1616-1622. doi:10.1016/j.fertnstert.2004.07.933 